Article

\title{
Electric Currents along Astrophysical Jets
}

\author{
Ioannis Contopoulos ${ }^{1,2}$ \\ 1 Research Center for Astronomy and Applied Mathematics, Academy of Athens, Athens 11527, Greece; \\ icontop@academyofathens.gr \\ 2 National Research Nuclear University (MEPhI), 31 Kashirskoe Highway, 115409 Moscow, Russia \\ Academic Editors: Emmanouil Angelakis, Markus Boettcher and Jose L. Gómez \\ Received: 14 July 2017; Accepted: 23 October 2017; Published: 25 October 2017
}

\begin{abstract}
Astrophysical black holes and their surrounding accretion disks are believed to be threaded by grand design helical magnetic fields. There is strong theoretical evidence that the main driver of their winds and jets is the Lorentz force generated by these fields and their associated electric currents. Several researchers have reported direct evidence for large scale electric currents along astrophysical jets. Quite unexpectedly, their directions are not random as would have been the case if the magnetic field were generated by a magnetohydrodynamic dynamo. Instead, in all kpc-scale detections, the inferred electric currents are found to flow away from the galactic nucleus. This unexpected break of symmetry suggests that a battery mechanism is operating around the central black hole. In the present article, we summarize observational evidence for the existence of large scale electric currents and their associated grand design helical magnetic fields in kpc-scale astrophysical jets. We also present recent results of general relativistic radiation magnetohydrodynamic simulations which show the action of the Cosmic Battery in the vicinity of astrophysical black holes.
\end{abstract}

Keywords: black hole physics; relativistic processes; magnetic fields

\section{Introduction}

Magnetic fields are an important constituent of cosmic plasmas at all astrophysical scales. They are thought to be produced by "seed" fields generated by random currents that are amplified by dynamo action in planetary and stellar interiors, accretion disks and generally rotating astrophysical plasmas. Whatever their origin, they are thought to be subsequently advected to large distances by stellar and galactic winds, and in fact direct observations, through Faraday rotation, polarization, Zeeman effect, etc., show a generally turbulent magnetic field structure on all astrophysical scales, from planets to galaxy clusters.

On the other hand, an important class of astrophysical structures, namely winds and jets from accretion disks around compact objects (primarily black holes), can most economically be explained through large scale grand design helical magnetic fields that extend from the immediate vicinity of the central compact object up to several million times larger scales [1-3]. This theoretical picture is supported by indirect observational evidence (AGN warm absorbers [4], Faraday RM gradients across astrophysical jets [5-7], polarization measurements [8], XRB Hardness-Intensity q-diagrams [9], GRB exponential afterglow decay [10], etc.), and by state-of-the-art MHD simulations [11]. In reality, magnetic fields in astrophysical jets are only partially ordered (as implied by a fairly high degree of detectable polarization), and often include a significant turbulent component. There is no unified opinion in the scientific community about whether the underlying magnetic field structure is indeed grand design helical, and a number of researchers are investigating other possible magnetic field configurations [12] and alternative sources for astrophysical jets (radiation pressure, hydrodynamics, collimation by an external medium, etc.). 
Here we argue that magnetic fields play a fundamental role in the generation, acceleration and collimation of astrophysical jets and winds, and that the observational evidence for the presence of large scale electric currents along kpc-scale astrophysical jets is convincing enough. In Section 2, we summarize the available observational evidence, and in Section 3, we briefly describe our own theory for the origin and geometric structure of the large scale magnetic field, the so-called Cosmic Battery. In Section 4, we present results of GR MHD simulations with radiation, and in Section 5, we conclude with our proposal for future research (theoretical, observational and numerical) that will further strengthen our theory for the origin and distribution of magnetic fields in astrophysical jets and winds.

\section{Observations of Electric Currents Along Kpc-Scale Astrophysical Jets}

Kronberg et al. [13] were the first to estimate a kpc-scale longitudinal electric current $\left(3 \times 10^{18} \mathrm{~A}\right)$ in the radio jet of an active galaxy (3C303). Their measurement was based on the detection of a gradient in the Faraday RM transverse to the jet axis in the barely resolved jet knot E3, and on a number of assumptions (estimates of the electron number density and the line-of-sight distance through the knot). Similar gradients consistent with longitudinal electric currents were observed on parsec scales $[14,15]$ long before [13], and many more such cases have been identified since (e.g., [6,16]). Their detection over kpc scales, though, yields strong support to the theoretical picture that grand design helical magnetic fields play a fundamental role in driving astrophysical jets, all the way from their origin in the galactic nucleus to their eventual demise in the radio lobes.

Christodoulou et al. [5] collected all radio sources they could find in the literature whose RM maps showed extended, monotonic, close to transverse RM gradients indicative of longitudinal electric currents on large (kiloparsec) scales, and also presented two new examples. Overall, out of the 89 published radio maps considered, they were able to identify large-scale monotonic transverse RM gradients (both firm and tentative) in 18 sources.

The latter result is by itself very important because it confirms the existence of large scale electric currents or equivalently large scale helical magnetic fields in astrophysical jets. What is more important though is that in all cases, the electric currents are observed to flow away from the galactic nucleus [17]. This unexpected break of symmetry implies that the large scale magnetic field in the jet cannot be generated by some random seed field amplified by an MHD dynamo process. The only obvious alternative is that the large scale magnetic field in the jet is generated by a battery process associated with the very origin of the jet around the central black hole. One compelling possibility is our own Cosmic Battery.

\section{The Cosmic Battery}

The Cosmic Battery is a simple physical mechanism that we proposed twenty years ago in order to justify the theoretical picture that large scale helical magnetic fields are an important constituent of astrophysical jets and winds of all scales [18]. According to it, the radiation drag on the plasma electrons at the inner edge of the accretion disk around the central black hole generates a toroidal (azimuthal) electric current in the direction of rotation which gives rise to poloidal (meridional) magnetic field loops around the inner edge of the disk. The outer footpoint of each loop resides well inside the accretion disk and diffuses outward on the local diffusion timescale (provided that this is shorter than the local accretion timescale). The inner footpoint is dragged inward by the accretion flow and eventually ends up near the black hole. Under these conditions, the magnetic flux that accumulates in the vicinity of the black hole points in the same direction as the angular velocity vector of the accretion disk ${ }^{1}$.

1 This statement is modified when the central black hole rotates at more than about $70 \%$ of maximal rotation. In that case, the inner edge of the accretion disk approaches close enough to the black hole horizon for the space-time rotation effect on 
The accretion disk drags the footpoints of these poloidal loops. As a result of differential rotation, the loops are wound, open up, and their inner part practically detaches from their outer part. When that happens, both parts of the formerly closed loops are wound backwards (opposite to the direction of rotation; see Figures 1 and 2 of [5]). The resulting wound field configuration corresponds to a large-scale axial electric current that flows toward the centre along the inner (with respect to the axis) jet spine, and a large-scale axial electric current that flows out from the centre along the outer (with respect to the axis) jet sheath. The central jet current closes along the interface between the inner and outer jets, whereas the outer jet electric current closes farther out, at the end of the jet. This configuration of the axial electric current predicted by $[5,18]$ is universal and unique to the Cosmic Battery. If we want to associate this with the observed symmetry breaking that we discussed in Section 2 above, we are compelled to associate the observed kpc-scale extragalactic jets with the above outer jet return field configuration. It is interesting that in several radio sources we observe a reversal of the direction of the axial electric current very close to the origin of the jet (sources marked with ${ }^{* \prime}$ in Table 1 of [5]). In all such cases, the inner/outer currents flow toward/away from the origin respectively. In the context of the Cosmic Battery, we are compelled to associate the observationally inferred reversed inward flowing electric current near the origin with the inner (spine) jet mentioned above $[7,20]$.

The above theoretical picture and observations are confirmed by GR radiation MHD simulations which show the growth of the magnetic field configuration predicted by the Cosmic Battery.

\section{GR Radiation MHD Simulations of the Cosmic Battery in Action}

The growth of the magnetic field $\mathbf{B}$ is governed by the induction equation which, in the presence of radiation, includes a new electric field source term equal to the radiation force $\mathbf{f}_{e}$ felt by the plasma electrons in the frame comoving with the plasma divided by the magnitude of the electron charge, namely

$$
d \mathbf{B} / d t=-c \nabla \times\left(\mathbf{E}+\mathbf{f}_{e} / e\right)
$$

E is the standard electric field expression, which includes both advection and diffusion terms. The inclusion of this term is straightforward in any numerical code that incorporates the dynamic effects of radiation, since the radiation pressure in the comoving frame is a fundamental quantity that is already calculated by the code. Of course, the programmer must decide whether their simulations apply to an electron-ion or to an electron-positron plasma².

We have performed in the past several numerical simulations of the Cosmic Battery process [18,21-23]. Our first simulations were performed in Newtonian spacetime and the source of radiation was considered to be isotropic. Koutsantoniou \& Contopoulos [19] then studied a more realistic radiation field with GR ray-tracing in an optically thick accretion flow around an astrophysical black hole in which the source of radiation is the accretion disk itself, and the radiation field in the comoving frame is distorted both by the disk and spacetime rotation. We have also performed the first fully GR, numerical simulations of the Cosmic Battery with the GR radiation MHD code KORAL [16]. These apply to a $10 M_{\odot}$ Schwarzschild black hole, but they can directly be generalized for a supermassive AGN black hole. The radiation field is generated by the optically thin accretion flow itself. The main result of our studies is that the Cosmic Battery is operating secularly (continuously) as predicted by [18], in parallel with the overall

photons to win over the aberration due to the orbital motion. When that takes place, the comoving azimuthal radiation pressure on the plasma electrons and the resulting azimuthal electric current and innermost poloidal magnetic field all reverse direction [19].

2 The Cosmic Battery operates in the accretion disk which consists of "light" electrons that feel the radiation pressure and "heavy" ions that do not. In an electron-positron plasma, there is no extra radiation term in the induction equation, and the Cosmic Battery would not operate. 
dynamics of the flow ${ }^{3}$. In all cases, from AGN to XRBs, it drives the system towards a MAD state (Magnetically Arrested Disk [24]; see Figure 1).
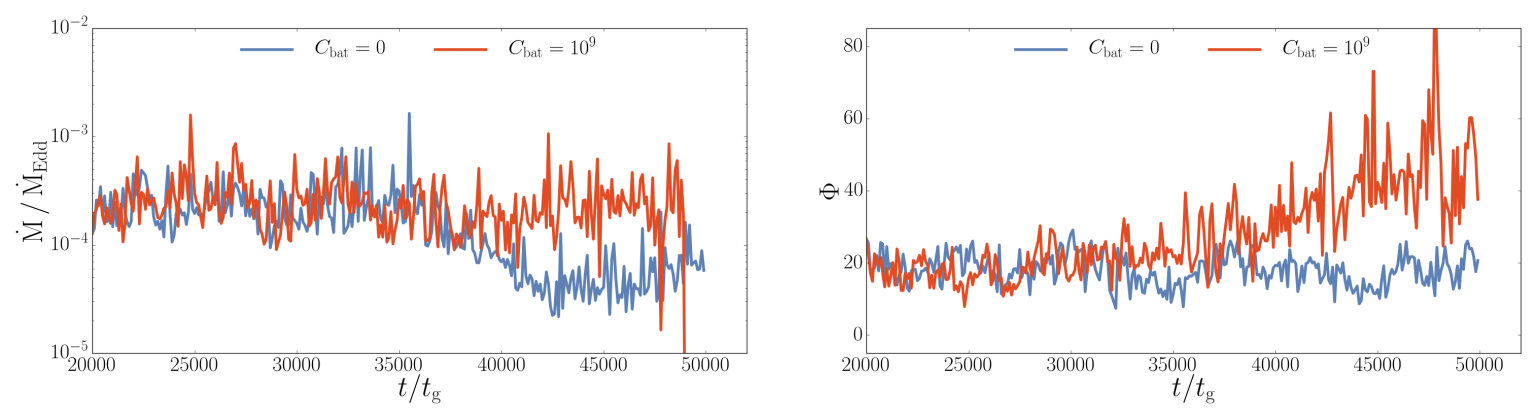

Figure 1. (Left) evolution of the mass accretion rate (in units of its Eddington value) in two 2D GR radiation MHD simulations, one with a random initial magnetic field (blue line), and one where the Cosmic Battery amplified by a factor of $10^{9}$ is introduced after 20,000 gravitation time scales $t_{g} \equiv G M / c^{3}$ (red line). After about 50,000 $t_{g}$ accretion stops (the black hole accretion rate $\dot{M}$ drops to zero) and a MAD state is reached. (Right) similarly for the magnetic flux $\Phi$ (in geometric units) threading the black hole horizon (from [16]).

The process is obviously very slow and the only practical way to observe it, within the available finite simulation time, is to artificially increase the Cosmic Battery source term in the induction equation by several orders of magnitude. This accelerates the growth of the magnetic field without distorting the action of the other forces acting in the system and, in particular, the radiation pressure. This can be realized clearly by the fact that the overall evolution of the accretion flow in Figure 1 remains indistinguishable from a standard evolution without the Cosmic Battery source term up to about 25,000 gravitational times after that term is switched on. Note that, as calculated in [18], the actual physical time scale for the manifestation of the effect of the Cosmic Battery is hundreds of millions of innermost gravitational times scales (in practice, these correspond to a few weeks in XRBs, and around one billion years in AGN).

\section{Future Research}

The Cosmic Battery in AGN and XRBs is a strong contender for the origin of magnetic fields in astrophysical jets and the Universe in general. More observations of RM gradients transverse to the axis of the jet are certainly needed. The expected improvement in resolution and sensitivity of the upcoming SKA radio telescope may be able to confirm, on the one hand, whether large scale electric currents are indeed present along astrophysical jets and, on the other hand, whether they all follow the universal flow pattern (inwards near the axis of the jet, outwards in its periphery) predicted by the Cosmic Battery.

The community should also invest in other types of observations which also give clues about the large scale structure of the magnetic field in astrophysical jets. For example, Donald Lynden-Bell looked at published intrinsic Faraday RM maps for the centres of galaxies seen at inclination angles of less than $60^{\circ}$ and found evidence that their central magnetic fields are preferentially oriented along the angular velocity vector of the galaxy (obtained from the direction of the galactic spirals), in accordance with the Cosmic Battery [25]. Other observations that require further investigation are asymmetric profiles of total intensity and polarization across the jets [26], magnetic field orientation changing from longitudinal to transverse [8], linear to circular polarization conversion [27], RM maps

3 GR effects become important only very close to the horizon of fast spinning black holes as discussed in the first footnote. Other than that, there are no significant differences between the results of full GR and earlier Newtonian simulations. 
from background sources around the jet [28,29], etc. The latter, in particular, are very significant since, according to the Cosmic Battery and observations reported in the present work, the large scale magnetic field that threads the kpc-scale jet consists of the return part of the magnetic field that threads the central black hole and possibly forms an electron-positron barely observable jet spine [30]. If this is the case, then according to the Cosmic Battery, the return field diffuses outward over large distances through the accretion disk. This implies that the observed kpc-scale jet probably constitutes the innermost (axially) part of the much more extended Magnetically Advected Winds-MAW reported in [31]. RM maps from background sources at large distances from the axis of the jet probe precisely these disk winds.

More numerical simulation work is also needed. We plan to extend our GR radiation MHD simulations in the case of a rotating black hole and for other types of accretion flows (optically thick, etc.). We also plan to investigate the reversal of the Cosmic Battery very close to the horizon of a fast rotating black hole discovered by [19].

Conflicts of Interest: The author declares no conflict of interest.

\section{Abbreviations}

The following abbreviations are used in this manuscript:

$\begin{array}{ll}\text { AGN } & \text { Active Galactic Nucleus } \\ \text { GR } & \text { General Relativity } \\ \text { GRB } & \text { Gamma-Ray Burst } \\ \text { MAD } & \text { Magnetically Arrested Disk } \\ \text { MAW } & \text { Magnetically Advected Wind } \\ \text { MHD } & \text { Magnetohydrodynamics } \\ \text { RM } & \text { Rotation Measure } \\ \text { XRB } & \text { X-Ray Binary }\end{array}$

\section{References}

1. Blandford, R.D.; Payne, D.G. Hydromagnetic flows from accretion discs and the production of radio jets. Mon. Not. R. Astron. Soc. 1982, 199, 883-903.

2. Contopoulos, J.; Lovelace, R.V.E. Magnetically driven jets and winds: exact solutions. Astrophys. J. 1994, 429, 139-152.

3. Fukumura, K.; Kazanas, D.; Shrader, C.; Behar, E.; Tombesi, F.; Contopoulos, I. Magnetic origin of black hole winds across the mass scale. Nat. Astron. 2017, 1, 62.

4. Fukumura, K.; Tombesi, F.; Kazanas, D.; Schrader, C.; Behar, E.; Contopoulos, I. Fast ionized X-ray absorbers in AGNs. Astron. Nachr. 2016, 337, 454-458.

5. Christodoulou, D.M.; Gabuzda, D.; Knuettel, S.; Contopoulos, I.; Kazanas, D.; Coughlan, C.P. Dominance of outflowing electric currents on decaparsec to kiloparsec scales in extragalactic jets. Astron. Astrophys. 2016, $591,61-71$.

6. Gabuzda, D.C.; Knuettel, S.; Reardon, B. Transverse Faraday-rotation gradients across the jets of 15 active galactic nuclei. Mon. Not. R. Astron. Soc. 2015, 450, 2441-2450.

7. Gabuzda, D.C.; Roche, N.; Kirwan, A.; Knuettel, S.; Nagle, M.; Houston, C. Parsec scale Faraday-rotation structure across the jets of nine active galactic nuclei. Mon. Not. R. Astron. Soc. 2017, 472, 1792-1801.

8. Murphy, E.; Gabuzda, D.; Cawthorne, T. Analysing the transverse structure of the relativistic jets of active galactic nuclei. Mon. Not. R. Astron. Soc. 2013, 430, 1504-1515.

9. Kylafis, N.D.; Contopoulos, I.; Kazanas, D.; Christodoulou, D.M. Formation and destruction of jets in X-ray binaries. Astron. Astrophys. 2012, 538, 5-10.

10. Nathanail, A.; Strantzalis, A.; Contopoulos, I. The rapid decay phase of the afterglow as the signature of the Blandford-Znajek mechanism. Mon. Not. R. Astron. Soc. 2016, 455, 4479-4486.

11. McKinney, J.C.; Tchekhovskoy, A.; Blandford, R.D. Alignment of Magnetized Accretion Disks and Relativistic Jets with Spinning Black Holes. Science 2013, 339, 49-52. 
12. Parfrey, K.; Giannios, D.; Beloborodov, A.M. Black hole jets without large-scale net magnetic flux. Mon. Not. R. Astron. Soc. 2015, 446, L61-L65.

13. Kronberg, P.P.; Lovelace, R.V.E.; Lapenta, G.; Colgate, S.A. Measurement of the Electric Current in a kpc-scale Jet. Astrophys. J. 2011, 741, L15-L18.

14. Asada, K.; Inoue, M.; Uchida, Y.; Kameno, S.; Fujisawa, K.; Iguchi, S. Mutsumi Mutoh A Helical Magnetic Field in the Jet of 3C273. Publ. Astron. Soc. Jpn. 2002, 54, L39-L43.

15. Gabuzda, D.C.; Murray, E.; Cronin, P. Helical magnetic fields associated with the relativistic jets of four BL Lac objects. Mon. Not. R. Astron. Soc. 2004, 351, L89-L93.

16. Contopoulos, I.; Nathanail, A.; Sadowski, A.; Kazanas, D.; Narayan, R. Numerical simulations of the Cosmic Battery in accretion flows around astrophysical black holes. Mon. Not. R. Astron. Soc. 2017, doi:10.1093/mnras/stx2249.

17. Knuettel, S.; Gabuzda, D.; O'Sullivan, S.P. Evidence for Toroidal B-Field Components in AGN Jets on Kiloparsec Scales. Galaxies 2017, 5, 61.

18. Contopoulos, I.; Kazanas, D. A Cosmic Battery. Astrophys. J. 1998, 508, 859-863.

19. Koutsantoniou, L.E.; Contopoulos, I. Accretion Disk Radiation Dynamics and the Cosmic Battery. Astrophys. J. 2014, 794, 27-39.

20. Lico, R.; Gomez, J.L.; Asada, K.; Fuentes, A. Interpreting the time variable RM observed in the core region of the TeV blazar Mrk 421. Mon. Not. R. Astron. Soc. 2017, 469, 1612-1616.

21. Christodoulou, D.M.; Contopoulos, I.; Kazanas, D. Simulations of the Poynting-Robertson Cosmic Battery in Resistive Accretion Disks. Astrophys. J. 2008, 674, 388-407.

22. Contopoulos, I.; Kazanas, D.; Christodoulou, D.M. The Cosmic Battery Revisited. Astrophys. J. 2006, 652, 1451-1456.

23. Contopoulos, I.; Nathanail, A.; Katsanikas, M. The Cosmic Battery in Astrophysical Accretion Disks. Astrophys. J. 2015, 805, 105-115.

24. Narayan, R.; Igumenshchev, I.V.; Abramowicz, M.A. Magnetically Arrested Disk: an Energetically Efficient Accretion Flow. Publ. Astron. Soc. Jpn. 2003, 55, L69-L72.

25. Lynden-Bell, D. Magnetism Along Spin. Observatory 2013, 133, $266-269$.

26. Gomez, J.L.; Marscher, A.P.; Jorstad, S.G.; Agudo, I.; Roca-Sogorb, M. Faraday Rotation and Polarization Gradients in the Jet of 3C120. Astrophys. J. 2008, 681, L69-L72.

27. Denise Carmen Gabuzda. Determining the Jet Poloidal B Field and Black-Hole Rotation Directions in AGNs. Galaxies 2017, submitted.

28. Feain, I.J.; Ekers, R.D.; Murphy, T.; Gaensler, B.M.; Macquart, J.-P.; Norris, R.P.; Cornwell, T.J.; Johnston-Hollitt, M.; Ott, J.; Middelberg, E. Faraday Rotation Structure on Kiloparsec Scales in the Radio Lobes of Centaurus A. Astrophys. J. 2009, 707, 114-125.

29. Pshirkov, M.S.; Tinyakov, P.G.; Kronberg, P.P.; Newton-McGee, K.J. Deriving the Global Structure of the Galactic Magnetic Field from Faraday Rotation Measures of Extragalactic Sources. Astrophys. J. 2011, 738, 192-205.

30. Asada, K.; Nakamura, M.; Pu, H.-Y. Indication of the Black Hole Powered Jet in M87 by VSOP Observations. Astrophys. J. 2016, 833, 56-62.

31. Contopoulos, I.; Kazanas, D.; Fukumura, K. Magnetically Advected Winds. Mon. Not. R. Astron. Soc. 2017, doi:10.1093/mnrasl/slx123.

(C) 2017 by the author. Licensee MDPI, Basel, Switzerland. This article is an open access article distributed under the terms and conditions of the Creative Commons Attribution (CC BY) license (http:/ / creativecommons.org/licenses/by/4.0/). 